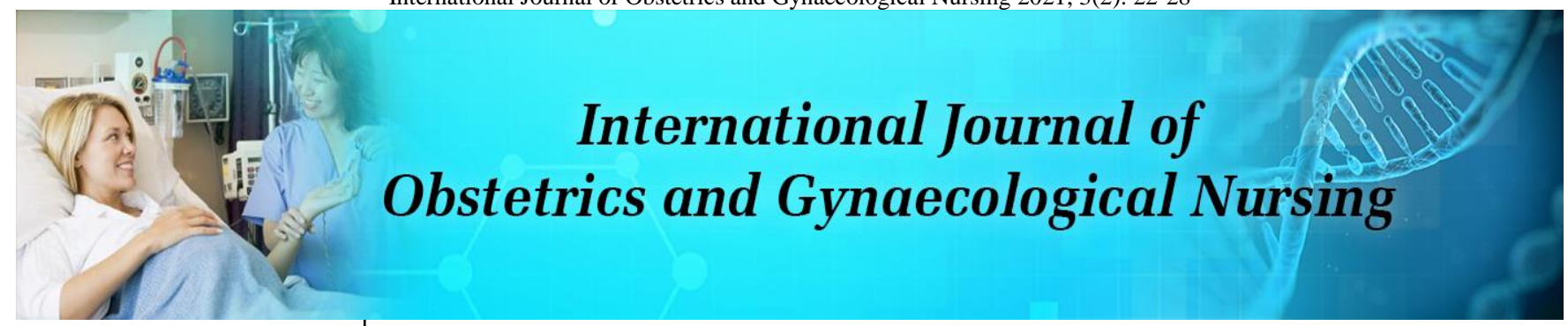

E-ISSN: 2664-2301 P-ISSN: 2664-2298 IJOGN 2021; 3(2): 22-28 Received: 12-05-2021 Accepted: 16-06-2021

Christiana Asiedu PhD, MPhil, MPH, Department of Adult Health, School of Nursing and Midwifery, College of Health and Allied Sciences, University of Cape Coast, Cape Coast, Ghana
Corresponding Author: Christiana Asiedu PhD, MPhil, MPH, Department of Adult Health, School of Nursing and Midwifery, College of Health and Allied Sciences, University of Cape Coast, Cape Coast, Ghana

\section{Assessment of impact of obstetric factors on use of skilled delivery service by pregnant women at health facilities in Ghana}

\section{Christiana Asiedu}

DOI: https://doi.org/10.33545/26642298.2021.v3.i2a.69

\begin{abstract}
Pregnancy and delivery complications account for greater proportion of deaths and disability among women in reproductive age than any other reproductive health problems. The purpose of the study was to assess the Assessment of Influence of Obstetric Variables $n$ he Use of Skilled Delivery Service by Pregnant Women at Health Facilities in Ghana. A cross-sectional design was utilised to achieve the overall objective of the study. The study covered pregnant women between the ages of 15-49 years in Central Region of Ghana. Questionnaire was designed and used as the main instrument for the study. Data collected were processed by the use of SPSS version 21 and analysed using descriptive and inferential statistics. Women with no history of miscarriage are 1.9 times more likely to use SDS compared to those who have had the experience of miscarriage in life $(\mathrm{OR}=1.94,95 \% \mathrm{CI}=1.34-2.83$, $p=<0.0001)$. Skilled birth attendants should educate the pregnant women about the complications associated with pregnancy to enable them seek timely care at health facilities.
\end{abstract}

Keywords: antenatal, complications, delivery, obstetric, pregnancy

\section{Introduction}

Achieving maternal health is measured by reduction of maternal mortality. From WHO's perspective, maternal death is the death of a woman while pregnant or within 42 days of termination of pregnancy, irrespective of the duration and the site of the pregnancy, from any cause related to or aggravated by the pregnancy or its management but not from accidental or incidental causes" ${ }^{[1,2]}$. Every woman in child bearing age (15-49 years) stands the risk of disability or death during pregnancy and delivery [3, 4, 5, 6, 7]. Pregnancy and delivery complications account for greater proportion of deaths and disability among women in reproductive age than any other reproductive health problems ${ }^{[8]}$.

From the WHO fact sheet report, globally, about 830 women die each day from preventable causes related to pregnancy and childbirth ${ }^{[6]}$. According to World Health Organization (WHO), global maternal mortality rate (MMR) has reduced by $44 \%$ between 1990 and 2015 [6]. That is from 523,000 in 1990 to 303, 000 maternal deaths in 2015. These maternal deaths mostly occur because of complications during pregnancy and childbirth ${ }^{[9]}$. The sad aspect is that these deaths could have been prevented with the use of skilled delivery service (SDS) during pregnancy, labour and during postnatal period. Almost all (99\%) of the maternal and newborn mortality occurs in the developing regions where resources for maternal health care is a major challenge. Sub-Saharan Africa (SSA) accounts for about $62 \%$ of global maternal deaths ${ }^{[9]}$. This could be attributed to the fact that about $53 \%$ of pregnant women in SSA still deliver without the assistance of skilled health personnel.

About one third of the global maternal deaths also happened in South Asia $(11 ; 9 ; 10)$. The maternal mortality ratio (MMR) in developing countries in 2015 was 239 per 100000 live births versus 12 per 100000 live births in developed countries ${ }^{[9]}$. The maternal deaths in the developing countries are about 14 times higher than that in developed countries. Maternal deaths account for $10 \%$ of all deaths among women in reproductive age ${ }^{[11]}$. There are large disparities between countries, but also within countries, and between women with high and low income and those women living in rural versus urban areas ${ }^{[11,9]}$. The MMR is the number of maternal deaths occurring in a given year per 100, 000 live births during the same period $[12,11,9]$. MMR is used to measure the risk of a woman dying once she is already pregnant. Collecting information and data on MMR and its rates is difficult and costly. 
This is because countries in SSA face challenges of quality health data management. In addition, the indicators used to measure MMR often do not register change over a short period, nor do they provide clear indications as to what actions should be developed to improve the situation. To address these challenges, proposals were made on the use of process indicators, which will enable skilled attendants to track the coverage over a specified period. These indicators include percentage of women who attend antenatal care; percentage of women who deliver in an institution; percentage of women who have a skilled attendant at birth; caesarian section rate and comprehensive and essential obstetric services per 500,000 population ${ }^{[9,12]}$.

The major causes that account for about $75 \%$ of all maternal mortality are severe bleeding, infection, unsafe abortion, eclampsia, obstructed labour, other direct causes and indirect causes (25\%) include anemia, malaria, malnutrition, violence, high risk pregnancy and infectious diseases ${ }^{[13]}$. Too many pregnancies, too short an interval between pregnancies, having a pregnancy too early in life, or having a pregnancy too late in life are also some of the identified factors which predispose women to pregnancy and delivery complications ${ }^{[14]}$. These factors most often affect women's health status negatively leading to birth injury, miscarriage, or stillbirth, anemia, fatigue, increased blood pressure, and decreased immunity to diseases such as malaria and reproductive tract infections ${ }^{[15]}$.

According to GSS the major causes of maternal death in most of the regions in Ghana are haemorrhage and hypertensive disorders ${ }^{[14]}$. The predisposing factors which lead to maternal deaths in the country are personal/ family/ community factors; delay in seeking care, inability to identify danger signs, poverty, lack of transportation in the community, and socio-cultural factors which prevent women from seeking health care at health facilities. Transport and communication challenges included nonmotorable roads, poor communication systems, inadequate number of ambulances in various regions, delayed response from ambulance service, and client paying for ambulance services ${ }^{[16]}$. In addition, inadequate availability and inappropriate distribution of essential logistics and supplies for essential obstetric care services are other factors that account for the maternal deaths in Ghana. Now, the Ghana Health Service (GHS) is embarking on evidence basedintervention. The findings of this study will add to the information available concerning the factors that determine the use of SDS in the country. This could enable the government of Ghana through ministry of health (MOH) and GHS address the challenges facing pregnant women in using SDS. The interventions which will be implemented by the government will lead to improvement in the use of maternal health services by pregnant women mostly SDS which will in turn reduce maternal mortality in the country. Poor health service provision by health personnel could lead to the death of women during pregnancy, labour, delivery and during postnatal period ${ }^{[16]}$. Behaviors and practices of health workers which could lead to maternal death includes poor assessment of pregnant women; poor collection and documentation of information about the pregnant women; improper management of pregnant women during labour and delivery; and negative attitude toward the pregnant women and among other things ${ }^{[16]}$. These factors could deter pregnant women from using SDS; and appears in an increased tendency of maternal and neonatal deaths ${ }^{[16]}$.
To prevent unexpected complications that are associated with pregnancy and delivery, quality maternal health services including SDS must be accessible and affordable to all women in child-bearing age ${ }^{[7]}$. To reduce MMR there is the need for mothers to have access to SDS during labour and delivery and emergency obstetric services. Ghana has made significant progress in increasing access and of maternal health services ${ }^{[17]}$. In Ghana, between 2013 and 2014 functional Community-Based Health Planning and Services (CHPS) zones were increased by $27 \%$ that is from 2,315 - 2,948 respectively ${ }^{[14]}$. Currently all the CHPS zones offer basic maternal health services and also make referrals of any obstetric emergencies to appropriate health facilities. Overall, about $47 \%$ out-patient services were provided by CHPS, sub-district clinics and health centers. These health facilities are very accessible to patients and clients mostly pregnant women. The training of midwives were also increased from 472 in 2011 to 1, 305 in $2014^{\text {[14]. }}$

Ghana was able to reduce MMR by $44 \%$ in 2014 . Statistics shows that MMR in the country was reduced from 740 per 100,000 live births in 1990 to 350 deaths per 100,000 live births in $2014{ }^{\text {[14] }}$. In 2014, Ghana was classified as making progress towards the achievement of MDG 5 by $2015^{[14]}$. The reducing trend fell short of the $5.5 \%$ annual decline required to achieve millennium development goal (MDG) 5 target of 185 per 100,000 by 2015 . This could be because of inadequate allocation of health resources to deserved communities as well as the neglect of some components in the fight against maternal mortality $[14,15]$. The failure of Ghana to achieve the MDG 5 stemmed from the low coverage of SDS (57\%). In a bid to find out the actual cause of failure, this study seeks to investigate the factors that accounted for the low utilization. The findings of the study inform maternal health policies and interventions that will enable majority if not all the pregnant women in Ghana to use SDS mostly at health facilities.

The lifetime risk of maternal death in Ghana was 1 in $66^{[14]}$. The lifetime risk is a measure of the probability of death over a woman's reproductive life. It assumes that most women have more than one pregnancy in their lifetime and is therefore a more realistic assessment of the risk an individual woman faces because of her reproductive capacity $[10,18,19]$.

\section{Purpose of the study}

The purpose of the study was to assess the Assessment of Influence of Obstetric Variables $n$ he Use of Skilled Delivery Service by Pregnant Women at Health Facilities in Ghana.

\section{Methods \\ Research Design}

The study was a descriptive cross-sectional survey that quantitatively explored the various factors that influence utilization of SDS in the Central Region of Ghana. The function of the research design is to ensure that the evidence obtained enables the researcher to answer the initial research objectives as unambiguously as possible. Obtaining relevant evidence entails specifying the type of evidence needed to answer the research objectives, to test a theory, to evaluate a programme or to accurately describe some phenomenon.

The strength of the descriptive design lies in the fact that it strives to confirm existing knowledge and that it is a flexible research design that provides an opportunity to examine all 
aspects of utilisation of SDS. The weaknesses of this study are that it is impossible either to establish causal relationships or to get reliable perspectives on the natural occurrence of a particular situation or the focus of interest of the study.

\section{Study Area}

The study focuses on the Central Region. The Central Region occupies an area of 9,826 square kilometers, which is about $6.6 \%$ of the total land area of Ghana. It is bounded on the south by the Gulf of Guinea and on the west by the Western Region. The region shares borders on the east with the Greater Accra Region, on the north with Ashanti Region and on the north-east with Eastern Region. The region has 20 administrative districts with the historical city of Cape Coast as the capital. About $63 \%$ of the region is rural. The population was estimated at 2,413,050 for the year 2013 with an annual growth rate of $3.1 \%{ }^{[14]}$ and a population density of about 215 inhabitants per square utilisation.

Out of the 20 districts, seven districts did not have district hospitals. These were Assin South, Gomoa East, Upper Denkyira West, Awutu senya, Awutu Senya East, Ekumfi and Agona East districts. The distribution of health facilities does not favour the large rural majority as most of the facilities are located in urban areas. This calls for a scaling up of the CHPS initiative to get basic services to the doorsteps of the people, especially those in rural areas. There are five health training institutions in the region located at Winneba, Cape Coast, Ankaful, Twifu Praso and Dunkwa-on-Offin.

\section{Population}

The target population for the study was pregnant women of reproductive age (WRA), between 15-49 years. Women in Reproductive Age who had delivered within the past three years prior to the study regardless of their birth outcome and those who had never given birth and are pregnant for the first time, in the Central Region of Ghana. Women in Reproductive Age between 15-49 years were used because the age bracket has been used by most studies, which were conducted about use of SDS globally. Women who gave birth outside the Central Region were excluded and if a woman gave birth twice within the 3 year period prior to the study, she was interviewed on the last delivery. Women who were below or beyond the reproductive age ( $15-49$ years) were also excluded from the study, as this age bracket is the main target for female reproductive years.

Women who were not pregnant, pregnant women who were not within the ages of $15-49$ years, those whose last birth were more than 3 years, those who gave birth 3 years prior to the study but outside Central Region and pregnant women who were not antenatal care attendees were excluded from the study.

\section{Sampling Procedure}

Sample size was 1100 pregnant women. The sample size determined was proportionally allotted to the health facilities selected based on their average monthly ANC attendance at each facility. The average number of clients was determined by considering the ANC attendance for the 3 months preceding the month of the study.

Multi-stage cluster sampling was used to select the respondents for the study. All the districts have health facilities. The districts were therefore grouped into two strata, urban and rural. The purposive sampling method was used to select public health facilities that provide ANC services within the selected districts such as hospitals, poly clinics, health centers and CHPS compounds. Simple random sampling technique was used to select 10 districts out of the 20 districts. The purposive sampling method was also used to select 1,000 women who had their last delivery between one and three years and 100 women who had never given birth.

The convenient sampling method was also used to select the pregnant women at each selected health facility for the study.

\section{Data collection instrument}

Questionnaire was used to collect the data from the respondents. The sub-sections of the questionnaire were on socio-demographic characteristics (section A) and obstetrical issues of respondents (Section B).

The "Sections B" of the questionnaire were four point Likert Scale of "Strongly Agree" (SA), "Agree" (A), "Disagree" (D), and "Strongly Disagree" (SD). All the responses for "agree" and "strongly agree" were re-categorised as "agree" while "disagree" and "strongly disagree" were recategorised as "disagree" for analysis.

In order to ensure validity, reliability and minimize errors of the instrument used for the data collection, a pre-test or preliminary trial of the instrument was conducted. The pretest was conducted at Effia Nkwanta hospital in Takoradi Metropolis in the Western Region to ensure clarity of the questions and to correct confusion over some items of the instrument before the actual fieldwork. The Effia Nkwanta hospital was selected because the pregnant women the hospital had the same background characteristics as the pregnant women in the Central Region of Ghana. The reliability of the items on the questionnaire were determined separately with the use of the Cronbach's alpha. This helped the researcher to determine how the items relate to each other. This helped to measure accuracy, trust worthiness and consistency and dependability of the instrument and the data collected. The Cronbach's Alpha for the instrument was 0.921 .

\section{Data collection procedure}

Data was collected mainly from primary source; directly from the responses obtained from the pregnant women. Ethical clearance for the study was sought from the University of Cape Coast Ethical Review Board and Ghana Health Service, Ethics Review Committee. Approval for the study was sought from the Central Regional Health Directorate and permission was sought from the District Health Directors, Medical Superintendents, and the incharges of the selected districts, health facilities and ANC clinics respectively.

The purpose of the study was explained to the clients. Six research assistants were trained and supported the data collection that is five Community Health Nursing trainees and one ANC clinic in-charge. The data were collected within 40 working days. That is from $4^{\text {th }}$ April $-31^{\text {st }}$ May, 2016. The sample size was divided by the 40 days, and approximately 25 clients answered the questionnaire at each clinic a day till the required number was obtained. Respondents were assured of confidentiality. 


\section{Data processing and analysis}

Data collected from respondents were processed using SPSS version 21. Descriptive and inferential statistics were used to analyse the data. The data collected were screened to ascertain the accuracy of the data, deal with missing data, and assess the effects of extreme values on the analysis.

The dependent variable, utilisation of SDS, was dichotomous; implying that a pregnant woman in labour would use SDS or not. There was the need to predict intention to use. This required predicting the probability of use or non-use. To determine the socio-demographic factors that predict use of SDS among pregnant women, logistic regression was conducted. This analytic technique produced results in the form of odds ratio, p-value, and confidence intervals, which were useful in discussions.

Logistic regression was the appropriate analysis to use because there was the need to analyse and predict the dependent variable which was a dichotomous outcome and categorical. There were only two categories of the independent variable; whether a pregnant woman would use SDS or not.

Descriptive statistics helped to summarize and describe the data. The frequencies provided information about the number of participants who responded to each item. Consideration was given to the dichotomous (whether client will use SDS at health facility or not) data.

\section{Ethical Considerations}

Ethical clearance for the study was sought from the University of Cape Coast Ethical Review Board and Ghana Health Service, Ethics Review Committee. Approval for the study was sought from the Central Regional Health Directorate and permission was also sought from the district/municipal health directors, hospital management and antenatal clinic in charges. Informed consent was sought from respondents before including them in the study. Participants who could not read and write were asked to thumb print as approval for informed consent after the purpose of the study has been explained to them and informed about their right to interrupt the interview at any time or decline from the study without any fear of future prejudice. This was achieved by giving them informed consent forms to fill. All information obtained from the participants were kept confidentially. The names of respondents were not associated with responses provided to ensure their anonymity. Participants were informed about their freedom to skip some of the questions and exit from the study. They were informed that the answering of the questionnaire will take 20 minutes.

\section{Results}

Multivariate analysis of obstetric variables including age of last child, gestational age, past experience of ever having miscarriages, ever had still birth/neonatal mortality and experience of complications during previous pregnancy /delivery was done.

Among multiparous pregnant women who did not intend to use SDS, those whose last child was 2 year of age were $46 \%$ $(\mathrm{n}=86)$. Among multiparous pregnant women who intended to use SDS, those whose last child was 2 year were $45 \%(\mathrm{n}=350)$ as presented in Table 1 .

Regarding the gestational age, among women who did not intend to use SDS, $45 \%(\mathrm{n}=100)$ of them were in their third trimester, (7-9 months) and also among women who intended to use SDS, $40 \%(\mathrm{n}=354)$ of them were in their third trimester, (7-9 months). Among the multigravida pregnant women who did not intend to use SDS, 68\% $(\mathrm{n}=$ 131) had ever had a miscarriage. Among the multigravida pregnant women who intended to use SDS, $80 \%(n=642)$ had ever had a miscarriage. Among the multigravida pregnant women who did not intend to use SDS, 89\% $(\mathrm{n}=$ 171) had ever had stillbirth/neonatal mortality. Among the multigravida pregnant women who intended to use SDS, $92 \%(n=743)$ had ever had still birth/neonatal mortality.

Regarding experience of pregnancy complications during previous pregnancy/delivery, $76 \%(\mathrm{n}=190)$ of those who did not intend to use SDS had ever had complications during pregnancy or delivery. Among those who intended to use SDS, 77\% $(n=619)$ had ever had complications during pregnancy or delivery. Results of multivariate analysis of obstetric variables and use of SDS are shown in Table 1.

Table 1: Frequency distribution of the obstetric variables

\begin{tabular}{|c|c|c|}
\hline Obstetric factors & No n (\%) & Yes n (\%) \\
\hline Age of last child (n=967) & & $18(2.3)$ \\
\hline Less than one year & $4(2.2)$ & $156(20.0)$ \\
\hline One year & $40(21.5)$ & $350(44.8)$ \\
\hline Two year & $86(46.2)$ & $257(32.9)$ \\
\hline Three year & $56(30.1)$ & $163(18.1)$ \\
\hline Gestational age (n=1100) & $32(14.6)$ & $364(41.3)$ \\
\hline 4-3 months & $87(39.7)$ & $354(40.2)$ \\
\hline 7-9 months & $100(45.7)$ & $642(79.8)$ \\
\hline Yes & & $163(20.2)$ \\
\hline No & $131(67.9)$ & \\
\hline Ever had a miscarriage in life (n=998) & $62(32.1)$ & \\
\hline Yes & & $743(92.2)$ \\
\hline Ever had stillbirth/neonatal mortality (n=980) & $63(7.8)$ \\
\hline Yes & $171(88.6)$ & $619(76.5)$ \\
\hline
\end{tabular}

Logistic regression analysis were done to ascertain the relative effect of obstetric variables (age of last child, gestational age, ever having a miscarriage in life, ever had still birth/neonatal mortality and whether the mother has had 
the experience of pregnancy complications during previous pregnancy/delivery) on use of SDS by pregnant women as shown in Table 2.

Overall the logistic regression model was significant at $2 \log 1=804.442 ;$ Nagelkerke $\mathrm{R}^{2}=.186 ; X^{2}=32.52 ; \mathrm{p}=$ .001 . The correct prediction rate was about $82.7 \%$. With the exception of having had miscarriage in life $(p<0.05)$, none of the obstetric variables investigated were found to be associated with the use of SDS. Women with no history of miscarriage were about 2 times more likely to use SDS as compared to those who have had the experience of miscarriage in life $(\mathrm{OR}=1.95,95 \% \mathrm{CI}=1.34-2.83, p=$ $<0.000)$. The effect of the obstetrics variables studied can be found in Table 2 .

Table 2: Influence of obstetric factors on SDS use

\begin{tabular}{|c|c|c|c|c|c|}
\hline \multirow{2}{*}{ Obstetric factors } & \multirow{2}{*}{$\mathbf{B}$} & \multirow{2}{*}{ Wald } & \multicolumn{3}{|c|}{ Use of SDS with current pregnancy } \\
\hline & & & OR & $95 \% \mathrm{CI}$ & $P$ value \\
\hline Age of last child $(n=967)$ & & 0.04 & & & 0.989 \\
\hline Less than one year & & & ref & & \\
\hline One year & -.052 & & 0.92 & $0.29-2.93$ & \\
\hline Two year & -.046 & & 0.95 & $0.31-2.94$ & \\
\hline Three year & .074 & & 1.00 & $0.32-3.11$ & \\
\hline Gestational age $(n=1100)$ & & 1.03 & & & 0.359 \\
\hline $1-3$ months & & & ref & & \\
\hline $4-6$ months & .257 & & 0.83 & $0.50-1.37$ & \\
\hline $7-9$ months & .041 & & 0.71 & $0.42-1.16$ & \\
\hline Ever had a miscarriage in life $(n=998)$ & & 3.50 & & & $<0.000$ \\
\hline Yes & & & ref & & \\
\hline No & .181 & & 1.95 & $1.34-2.83$ & \\
\hline Ever had stillbirth/neonatal mortality $(\mathrm{n}=980)$ & & -1.29 & & & 0.197 \\
\hline No & & & ref & & \\
\hline Yes & .181 & & 0.70 & $0.41-1.20$ & \\
\hline Complications during previous pregnancy/delivery $(\mathrm{n}=980)$ & & 0.58 & & & 0.563 \\
\hline No & & & ref & & \\
\hline Yes & .827 & & 1.13 & $0.75-1.68$ & \\
\hline
\end{tabular}

OR: odds ratio, CI: confidence interval, Ref: reference category

Table 3: Cross tabulations and Chi-Square of Background Characteristics and Obstetric Complications

\begin{tabular}{|c|c|c|c|c|c|c|c|}
\hline \multirow{3}{*}{ Demographic variables } & \multicolumn{6}{|c|}{ Obstetric complications } & \\
\hline & \multicolumn{2}{|c|}{ Ever had miscarriage } & \multicolumn{2}{|c|}{ Still birth/neonatal death } & \multicolumn{2}{|c|}{ Complications } & \\
\hline & No N (\%) & Yes N (\%) & No & Yes & No & Yes & \\
\hline Age in years & 0.029 & & 0.001 & & 0.181 & & p-value \\
\hline Less the 20 & $46(85.2)$ & $8(14.8)$ & $54(100)$ & $0(0.0)$ & $46(85.2)$ & $8(14.8)$ & \\
\hline $20-29$ & $437(80)$ & $109(20)$ & 507(92.7) & $40(7.3)$ & $421(77.5)$ & $122(22.5)$ & \\
\hline $20-39$ & $265(73.2)$ & $97(26.8)$ & $325(89.8)$ & $37(10.2)$ & $258(74.1)$ & $90(25.9)$ & \\
\hline $40-49$ & $25(69.4)$ & $11(30.6)$ & $28(77.8)$ & $8(22.2)$ & $24(68.6)$ & $11(31.4)$ & \\
\hline Marital status & 0.561 & & 0.924 & & 0.266 & & p-value \\
\hline Never married & $62(79.5)$ & $16(20.5)$ & $71(91)$ & $7(9.0)$ & $58(77.3)$ & $17(22.7)$ & \\
\hline Married & $546(78.1)$ & $153(21.9)$ & $643(91.9)$ & $57(8.1)$ & $514(75.4)$ & $168(24.6)$ & \\
\hline Cohabiting & $158(74.9)$ & $53(25.1)$ & $195(92.4)$ & $16(7.6)$ & $172(80.8)$ & $41(19.2)$ & \\
\hline Level of formal education & 0.016 & & 0.759 & & 0.689 & & p-value \\
\hline No formal edu & $115(68.9)$ & $52(31.1)$ & $151(90.4)$ & $16(9.6)$ & $123(73.2)$ & $45(26.8)$ & \\
\hline Basic edu & $464(78.0)$ & $131(22.0)$ & $543(91.3)$ & $52(8.7)$ & $445(77.5)$ & $129(22.5)$ & \\
\hline Secondary edu & $110(81.5)$ & $25(18.5)$ & $125(91.9)$ & $11(8.1)$ & $106(76.8)$ & $32(23.2)$ & \\
\hline Tertiary edu & $84(83.2)$ & $17(16.8)$ & $95(94.1)$ & $6(5.9)$ & $75(75)$ & $25(25)$ & \\
\hline Main Occupation & 0.422 & & 0.951 & & 0.299 & & p-value \\
\hline Self employed & 593(76.6) & $181(23.4)$ & $707(91.3)$ & $67(8.7)$ & $583(76.7)$ & $177(23.3)$ & \\
\hline Civil servant & $91(82)$ & $20(18)$ & $102(91.9)$ & $9(8.1)$ & $76(71.0)$ & $31(29.0)$ & \\
\hline Unemployed & $89(78.8)$ & $24(21.2)$ & $105(92.1)$ & $9(7.9)$ & $90(79.6)$ & $23(20.4)$ & \\
\hline
\end{tabular}

Cross tabulations and chi-square of background characteristics and obstetric complications analysis were done. Concerning the age of the pregnant women and ever had miscarriage, majority of the women between 40-49 years has had a miscarriage $(31 \% ; n=11)$, still birth/neonatal death $(22 \% ; \mathrm{n}=8)$, and complications during previous pregnancy/delivery $(31 \% ; n=11)$. Regarding marital status and obstetric complications, miscarriage was common among women who were cohabiting (25\%; $n=53)$, and about $25 \%$ of the married women had ever had complications during pregnancy/delivery. Majority of the women who ever had miscarriage and complications were women with no formal education, miscarriage $31 \% \quad(n=52$ and) complication during pregnancy/delivery $37 \%(n=45)$. Furthermore, concerning main occupation of the respondents, women who were self-employed were the majority that had ever experienced miscarriage (24\%; $\mathrm{n}=181$ ) and among the women had ever experience complications during pregnancy and delivery, civil servant women were the majority $(29 \% ; \mathrm{n}=31)$ as presented in Table 3.

The chi-square analysis also suggested that the age of the 
women and their level of education had influence on their obstetric complications, Table 3 .

\section{Discussion}

The study did not find a statistically significant effect of previous history of birth complication and SDS use which replicates the findings of Potter who showed that prolonged labour or bleeding is not associated with SDS ${ }^{[20]}$. It is generally expected that mothers who have had the experience of neonatal deaths, loss of a new-born baby, loss of female relative during child birth and other birth complications during previous deliveries would make women mindful of the risks of childbirth and the importance of SDS. This should increase the probability of using SDS for subsequent deliveries. Women with specific medical interventions in a previous delivery, for instance a caesarean section, will be encouraged by health workers to seek skilled care for subsequent deliveries since there is an increased risk for rupture with a scarred uterus [21]. Complications during an attempted home delivery often influence women and their families to seek professional care, even though the original intention was to deliver at home.

The result of no significant effect of previous history of birth complication and neonatal mortality could be attributed to the fact that only few women in the current study indicated that they have had birth complications or experienced neonatal mortality before $(<9 \%$ for neonatal and $<23 \%$ for birth complication). Since majority of these women had never experienced complication during delivery, there were no compelling reasons to influence their choice of place of delivery for the index birth. This study also shows that a higher proportion of women attended ANC which could reduce the propensity of future birth complications and neonatal mortality as a result of the education they received during ANC period. Drastic reduction of reported birth complication and neonatal mortality in Central Region showed that there may be no enthralling reasons why complications and neonatal mortality could lead to future SDS use. The results contradict previous complex surveys that used logistic

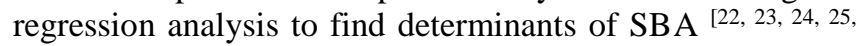
26, 27].

None of the studies reviewed directly assessed the impact of previous history of miscarriage on SDS use. The causes of miscarriage or spontaneous abortion are easily identified by trained medical staff in facilities especially when the woman attend ANC. It is natural to believe that mothers who have never experienced miscarriage may be well informed, have limited biological deficiencies or strong immune system, educated and understand the importance of ANC and SDS. These mothers may attribute their success to previous ANC attendance and facility delivery and that could have influenced their choice of intended SDS use for the index birth.

The age of last child to the index birth (birth spacing) was not found to be related to SDS use which does not support the findings of studies done in Nepal and India ${ }^{[28,29]}$. It is known that women in their first pregnancy are more likely to have their babies delivered in health facility especially when the woman really wanted to be pregnant for the first time. Poorly spaced pregnancies have been documented worldwide to result in adverse maternal and child health outcomes ${ }^{[30]}$. The reason could be that short birth intervals are generally associated with health risk knowledge, information availability, religion, poverty, education and use of contraceptive. These factors are also found to be related to facility delivery. The WHO recommends a minimum inter-birth interval of 33 months between two consecutive live births in order to reduce the risk of adverse maternal and child health outcomes ${ }^{[8]}$. This study did not find significant differences in the proportion of facility delivery among birth spacing categories because it is independent of facility delivery which is basically free in Ghana coupled with the fact that the mother may have valid NHIS membership card. The consequences of poorly spaced pregnancies indicated by WHO may not be a key deterrent among women who are not well educated and are unwilling to use contraceptives with the firm conviction that after all, having SDS at birth is virtually free. This reason might have contributed to why this study could not really distinguish between mothers who are very much aware of the risk of poor birth spacing and those who are not. Almost all the mothers independent of the age of their last child intended to use SDS.

The findings of the study is in line with the Andersen's Behavioural Model of Health Services Utilisation. According to the model need factors (illness variables and response variables) must be considered when thinking about use of any health service ${ }^{[31]}$. For pregnant women to use SDS, there must first be a need to use that service. The need to use SDS by the pregnant women who had never had miscarriage in life is to prevent such negative outcome of pregnancy and delivery and any morbidity, mortality and disability associated with pregnancy.

\section{Conclusion}

Health care providers must intensify health education and counselling to enable pregnant women to identify their health care needs, which will enable them to use SDS. SDS at health facilities must meet quality standards to prevent women and their babies from complications to motivate other pregnant women to use such health service when the need arises.

\section{Acknowledgements}

I acknowledge all my mentors. I am highly indebted to various writers from whom references were made. I would like to thank all other individuals who provided the needed support for me to complete this study.

\section{References}

1. Abou Zahr C. Global burden of maternal death and disability. British Medical Bulletin 2003;67:1-11.

2. World Health Organisation. Trends in Maternal Mortality: 1990 to 2010: Estimates Developed by WHO, UNICEF, UNFPA and the World Bank. Geneva: World health organisation 2012.

3. Bhutta ZA, Black RE. Global maternal, new born, and child health - so near and yet so far. New England Journal of Medicine 2013;369:23, 2226-2235.

4. Say L, Chou D, Gemmill A, Tunçalp Ö, Moller AB, Daniels $\mathrm{J}$ et al. Global causes of maternal death: A WHO systematic analysis. The Lancet Global Health 2014;2:6, e323-e333.

5. United Nations Children's Fund (UNICEF). The state of the world's children 2009: Maternal and New born Health. New York: Author 2008. 
6. World Health Organisation. World Health Statistics 2016: Monitoring Health for the SDGs Sustainable Development Goals. World Health Organisation. Geneva: Author 2016a.

7. World Health Organisation. Women and health: today's evidence tomorrow's agenda. Geneva: World Health Organisation 2009.

8. World Health Organisation. Classifying health workers. Geneva: WHO 2010.

9. World Health Organisation. Global, regional, and national levels and trends in maternal mortality between 1990 and 2015, with scenario-based projections to 2030: A systematic analysis by the UN maternal mortality estimation inter-agency group. Geneva: Author 2016b.

10. World Health Organisation, UNICEF. Trends in maternal mortality: 1990 to 2013: estimates by WHO, UNICEF, UNFPA, The World Bank and the United Nations Population Division: executive summary. Geneva: Author 2014.

11. Wang P, Connor AL, Guo E, Nambao M, Chanda- Kapata $\mathrm{P}$, Lambo $\mathrm{N}$ et al. Measuring the impact of non- monetary incentives on facility delivery in rural Zambia: A clustered randomised controlled trial. Tropical Medicine \& International Health 2016;21(4):515-524.

12. Penn-Kekana L, Blaauw D. A rapid appraisal of maternal health services in South Africa: A health systems approach. Johannesburg: Centre for Health Policy, University of the Witwatersrand. Health Systems Development Programme 2002.

13. World Health Organisation. World Health statistics. Geneva 2014.

14. Ghana Statistical Service (GSS). Ghana Health Service (GHS), ICF International, Ghana Demographic and Health Survey 2014: Key indicators report. Maryland: Authors 2015.

15. United Nations Children's Fund (UNICEF). Generation 2030 Africa, Child Demographics in Africa. Division of Data, Research, and Policy. New York: Author 2014.

16. Ghana Health Service (GHS). Holistic assessment of the health sector programme of work 2013 Ghana. Accra: Ministry of Health 2014.

17. Kruk ME, Freedman LP. Healthcare financing and utilization of maternal health services in developing countries. Health Policy and Planning 2007;22:303310 .

18. Wilmoth J. The lifetime risk of maternal mortality: concept and measurement. Bulletin of the World Health Organisation 2009;87(4):256-262.

19. Zahr CA, Wardlaw TM, Choi Y. Maternal mortality in 2000: estimates developed by WHO, UNICEF and UNFPA. World Health Organisation. Geneva: WHO 2004.

20. Potter JE. Utilización de los servicios de salud materna en el México rural. Salud Pública de México 1988;30(3):387-402.

21. Gabrysch S, Campbell OMR. Still too far to walk: Literature review of the determinants of delivery service use. BMC Pregnancy and Childbirth 2009;9(1):34. https://doi.org/10.1186/1471-2393-9-34.

22. Afsana K, Rashid SF. The challenges of meeting rural Bangladeshi women's needs in delivery care. Reproductive Health Matters 2001;9(18):79-89.
23. Bloom SS, Wypij D, Das Gupta M. Dimensions of women's autonomy and the influence on maternal health care utilization in a north Indian city. Demography 2001;38(1):67-78.

24. Burgard S. Race and pregnancy-related care in Brazil and South Africa. Social Science \& Medicine 2004;59(6):1127-1146.

25. Mesko N, Osrin D, Tamang S, Shrestha BP, Manandhar DS, Manandhar $\mathrm{M}$ et al. Care for perinatal illness in rural Nepal: A descriptive study with cross-sectional and qualitative components. BMC International Health and Human Rights 2003;3(1):3. https://www.ncbi.nlm.nih.gov/pubmed/12932300.

26. Pomeroy AM, Koblinsky M, Alva S. Who gives birth in private facilities in Asia? A look at six countries. Health Policy Plan 2014;29(1):i38-i47.

27. Telfer ML, Rowley JT, Walraven GE. Experiences of mothers with antenatal, delivery and postpartum care in rural Gambia. African Journal of Reproductive Health 2002;6:74-83.

28. Stephenson R, Tsui AO. Contextual influences on reproductive health service use in Uttar Pradesh, India. Studies in Family Planning 2002;33(4):309-320.

29. Sharma SK, Sawangdee Y, Sirirassamee B. Access to health: Women's status and utilization of maternal health services in Nepal. Journal of Biosocial Science 2007;39(5):671-692.

30. Exavery A, Mrema S, Shamte A, Bietsch K, Mosha D, Mbaruku G et al. Levels and correlates of nonadherence to WHO recommended inter-birth intervals in Rufiji, Tanzania. BMC Pregnancy and Childbirth 2012;12(1):152. Doi: 10.1186/1471-2393-12-152.

31. Andersen R, Newman JF. Societal and individual determinants of medical care utilization in the United States. Milbank Quarterly 2005;83:4. 10.1111/j.14680009.2005.00428. 